\title{
New Types of Fuzzy Filter on Lattice Implication Algebras
}

\author{
Yi Liu (Corresponding author) \\ College of Mathematics and Information Science, Neijiang Normal University \\ Neijiang, Sichuan 641112, China \\ Tel: 86-832-234-3417Ｅ-mail: liuyiyl@live.cn
}

Yang Xu

Intelligent Control Development Center, Southwest Jiaotong University

Chengdu, Sichuan 610031, China

Tel: 86-28-8760-0760Ｅ-mail: xuyang@home.swjtu.edu.cn

This work is supported by National Natural Science Foundation of P.R.China (Grant no. 60875034).

\begin{abstract}
Extending the belongs to $(\epsilon)$ relation and quasi-coincidence with $(q)$ relation between fuzzy points and a fuzzy subsets, the concept of $(\alpha, \beta)$-fuzzy filters and $(\bar{\alpha}, \bar{\beta})$-fuzzy filters of lattice implication algebras are introduced, where $\alpha, \beta \in\left\{\in_{h}\right.$ $\left., q_{\delta}, \epsilon_{h} \vee q_{\delta}, \epsilon_{h} \wedge q_{\delta}\right\}, \bar{\alpha}, \bar{\beta} \in\left\{\overline{\epsilon_{h}}, \overline{q_{\delta}}, \overline{\epsilon_{h}} \vee \overline{q_{\delta}}, \overline{\epsilon_{h}} \wedge \overline{q_{\delta}}\right\}$ but $\alpha \neq \epsilon_{h} \wedge q_{\delta}, \bar{\alpha} \neq \overline{\epsilon_{h}} \wedge \overline{q_{\delta}}$, respectively, and some related properties are investigated. Some equivalent characterizations of these generalized fuzzy filters are derived. Finally, the relations among these generalized fuzzy filters are discussed. Special attention to $\left(\epsilon_{h}, \epsilon_{h} \vee q_{\delta}\right)$-fuzzy filter and $\left(\overline{\epsilon_{h}}, \overline{\epsilon_{h}} \vee \overline{q_{\delta}}\right)$-fuzzy filter are generalizations of $(\in, \in \vee q)$-fuzzy filter and $(\bar{\epsilon}, \bar{\epsilon} \vee \bar{q})$-fuzzy filter, respectively.
\end{abstract}

Keywords: Lattice implication algebras, Fuzzy filter, $(\alpha, \beta)$-fuzzy filter, $(\bar{\alpha}, \bar{\beta})$-fuzzy filter

\section{Introduction}

Intelligent information processing is one important research direction in artificial intelligence. Information processing dealing with certain information is based on the classical logic. However, non-classical logics including logics behind fuzzy reasoning handle information with various facets of uncertainty such as fuzziness, randomness, etc. Therefore, nonclassical logic have become as a formal and useful tool for computer science to deal with uncertain information. Manyvalued logic, a great extension and development of classical logic, has always been a crucial direction in non-classical logic. In the field of many-valued logic, lattice-valued logic plays an important role for the following two aspects: One is that it extends the chain-type truth-valued field of some well known present logic to some relatively general lattice. The other is that the incompletely comparable property of truth value characterized by general lattice can more efficiently reflect the uncertainty of human being's thinking, judging and decision. Hence, lattice-valued logic is becoming an active research field which strongly influences the development of algebraic logic, computer science and artificial intelligent technology. In order to provide a reliable logical foundation for uncertain information processing theory, especially for the fuzziness, the incomparability in uncertain information in the reasoning, and, establish a logical system with truth value in a relatively general lattice. Combining algebraic lattice and implication algebra, $\mathrm{Xu}(\mathrm{Xu}, 1993)$ proposed the concept of lattice implication algebras(LIA for short) and discussed some of it's properties. Since then this logical algebra has been extensively investigated by several researchers(Y.Xu,1993, etc). Recently, Jun et al(Y.B.Jun,2007) introduced the concept of $(\in, \in \vee q)$-fuzzy implicative filter of a lattice implication algebra. Zhan et al(J.M.Zhan,2009) further to investigated this kind of fuzzy implicative filters.

The concept of fuzzy set was introduced by Zadeh(Zadeh, 1965). Rosenfeld inspired the fuzzification of algebraic structure and introduced the notion of fuzzy subgroup (Rosenfeld, 1971). The idea of fuzzy point and 'belongingness ' and 'quasi-coincidence' with a fuzzy set were given by $\mathrm{Pu}$ and Liu(P.M,Pu, 1980). A new type of fuzzy subgroup (viz $(\epsilon, \in \vee q)$-fuzzy subgroup) was introduced(S.K.Bhakat,1996). In fact, $(\epsilon, \in \vee q)$-fuzzy subgroup is an important and useful generalization of Rosenfeld's fuzzy subgroup. The idea of fuzzy point and 'belongingness ' and 'quasi-coincidence ' with a fuzzy set have been applied some important algebraic system(S.K.Bhakat,1999, etc).

This paper, as a continuation of (Y.B.Jun,2007, J.M.Zhan,2009), we extend the concept of quasi-coincidence and further to investigate the $(\in, \in \vee q)$-fuzzy filters, proposing the concept of $\left(\epsilon_{h}, \epsilon_{h} \vee q_{\delta}\right)$-fuzzy filter and $\left(\overline{\epsilon_{h}}, \overline{\epsilon_{h}} \vee \overline{q_{\delta}}\right)$-fuzzy filter. We investigate relations between $\left(\epsilon_{h} \vee q_{\delta}, \epsilon_{h} \vee q_{\delta}\right)\left(\left(\overline{\epsilon_{h}}, \overline{\epsilon_{h}} \vee \overline{q_{\delta}}\right)\right)$-fuzzy filters $\left(\epsilon_{h}, \epsilon_{h} \vee q_{\delta}\right)\left(\left(\overline{\epsilon_{h}}, \overline{\epsilon_{h}} \vee \overline{q_{\delta}}\right)\right)$-fuzzy filters, filters of $\mathscr{L}$. We establish characterizations of $\left(\epsilon_{h}, \epsilon_{h} \vee q_{\delta}\right)\left(\left(\overline{\epsilon_{h}}, \overline{\epsilon_{h}} \vee \overline{q_{\delta}}\right)\right)$-fuzzy filters and give some equivalent conditions of $\left(\epsilon_{h}, \epsilon_{h} \vee q_{\delta}\right)\left(\left(\overline{\epsilon_{h}}, \overline{\epsilon_{h}} \vee \overline{q_{\delta}}\right)\right)$-fuzzy filters. Of course, we can discuss $(\alpha, \beta)$-fuzzy (implicative, ultra-, associative) filter in the same way. It will be of great use to provide theoretical foundation to design intelligent information processing systems.

In this paper, denote $\mathscr{L}$ as a lattice implication algebra $\left(L, \vee, \wedge,{ }^{\prime}, \rightarrow, O, I\right)$. 


\section{Preliminaries}

Let $(L, \vee, \wedge, O, I)$ be a bounded lattice with an order-reversing involution ', the greatest element $I$ and the smallest element $O$, and $\rightarrow: L \times L \longrightarrow L$ be a mapping. $\mathscr{L}=\left(L, \vee, \wedge,{ }^{\prime}, \rightarrow, O, I\right)$ is called a lattice implication algebra if the following conditions hold for any $x, y, z \in L$ :

$\left(I_{1}\right) x \rightarrow(y \rightarrow z)=y \rightarrow(x \rightarrow z)$.

$\left(I_{2}\right) x \rightarrow x=I$.

(I $) x \rightarrow y=y^{\prime} \rightarrow x^{\prime}$.

(I $\left.I_{4}\right) \rightarrow y=y \rightarrow x=I$ implies $x=y$.

$\left(I_{5}\right)(x \rightarrow y) \rightarrow y=(y \rightarrow x) \rightarrow x$.

$\left(l_{1}\right)(x \vee y) \rightarrow z=(x \rightarrow z) \wedge(y \rightarrow z)$.

$\left(l_{2}\right)(x \wedge y) \rightarrow z=(x \rightarrow z) \vee(y \rightarrow z)$.

In this paper, denote $\mathscr{L}$ as lattice implication algebra $\left(L, \vee, \wedge,{ }^{\prime}, \rightarrow, O, I\right)$.

We list some basic properties of lattice implication algebras. It is useful to develop these topics in other sections.

Let $\mathscr{L}$ be a lattice implication algebra. Then for any $x, y, z \in L$, the following conclusions hold:

(1) if $I \rightarrow x=I$, then $x=I$.

(2) $I \rightarrow x=x$ and $x \rightarrow O=x^{\prime}$.

(3) $O \rightarrow x=I$ and $x \rightarrow I=I$.

(4) $(x \rightarrow y) \rightarrow((y \rightarrow z) \rightarrow(x \rightarrow z))=I$.

(5) $(x \rightarrow y) \vee(y \rightarrow x)=I$.

(6) if $x \leq y$, then $x \rightarrow z \geq y \rightarrow z$ and $z \rightarrow x \leq z \rightarrow y$.

(7) $x \leq y$ if and only if $x \rightarrow y=I$.

(8) $(z \rightarrow x) \rightarrow(z \rightarrow y)=(x \wedge z) \rightarrow y=(x \rightarrow z) \rightarrow(x \rightarrow y)$.

(9) $x \rightarrow(y \rightarrow z)=(x \vee y) \rightarrow z$ if and only if $x \rightarrow(y \rightarrow z)=x \rightarrow z=y \rightarrow z$.

(10) $z \leq y \rightarrow x$ if and only if $y \leq z \rightarrow x$.

A non-empty subset $F$ of a lattice implication algebra $\mathscr{L}$ is called a filter of $\mathscr{L}$ if it satisfies

(F1) $I \in F$.

(F2) $(\forall x \in F)(\forall y \in L)(x \rightarrow y \in F \Rightarrow y \in F)$.

A fuzzy subset of a nonempty set $X$ is defined as a mapping from $\mathrm{X}$ to $[0,1]$, where $[0,1]$ is the usual interval of real numbers.

A fuzzy subset $A$ of $\mathscr{L}$ is said to be a fuzzy filter if

$(\mathrm{F} 3)(\forall x \in L)(A(I) \geq A(x))$.

$(\mathrm{F} 4)(\forall x, y \in L)(A(y) \geq \min \{A(x), A(x \rightarrow y)\})$.

A level set of a fuzzy set $A$ in $\mathscr{L}$ is the set

$U(A ; \alpha):=\{x \in L \mid A(x) \geq \alpha\}, \alpha \in[0,1]$.

A fuzzy set $A$ of a lattice implication algebra $\mathscr{L}$ of the form

$$
A(y)= \begin{cases}t \in(0,1] & \text { if } y=x, \\ 0 & \text { otherwise } .\end{cases}
$$

is said to be a fuzzy point with support $x$ and value $t$ and is denoted by $x_{t}$. A fuzzy point $x_{t}$ is said to belong to (resp. be quasi-coincident with) a fuzzy set $A$, written as $x_{t} \in A$ (resp. $\left.x_{t} q A\right)$ if $A(x) \geq t$ (resp. $\left.A(x)+t>1\right)$. If $x_{t} \in A$ or (resp. and) $x_{t} q A$, then we write $x_{t} \in \vee q A$. The symbol $\overline{\epsilon \vee q}$ means $\in \vee q$ doesn't hold. Using the notion of ' belongingness $(\in)^{\prime}$ and 'quasi-coincidence $(q)^{\prime}$ ' of fuzzy point with fuzzy subsets, the concept of $(\alpha, \beta)$-fuzzy sub semigroup, where $\alpha$ and $\beta$ are any one of $\{\in, \in \vee q, \in \wedge q\}$ with $\alpha \neq \in \wedge q$, was introduced in (S.K.Bhakat,1996). It is worthy to note that the most viable generalization of Rosenfeld's fuzzy subgroup is the notion of $(\in, \in \vee q)$-fuzzy subgroup. The detailed research with $(\in, \in \vee q)$-fuzzy subgroup has been considered in(S.K.Bhakat, 1996). 


\section{3. $(\alpha, \beta)$-fuzzy filters}

In this section, we first to extend the concept of quasi-coincidence. In what follows, we let $h, \delta \in[0,1]$ be such that $h<\delta$ and $r \in(h, 1]$. For a fuzzy point $x_{r}$ and a fuzzy subset $A$ on $\mathscr{L}$, we say that

(1) $x_{r} \in_{h} A$ if $A(x) \geq r>h$.

(2) $x_{r} q_{\delta} A$ if $A(x)+r>2 \delta$.

(3) $x_{r} \in_{h} \vee q_{\delta} A$ if $x_{r} \in_{h} A$ or $x_{r} q_{\delta} A$.

(4) $x_{r} \in_{h} \wedge q_{\delta} A$ if $x_{r} \in_{h} A$ and $x_{r} q_{\delta} A$.

(5) $x_{r} \bar{\alpha} A$ if $x_{r} \alpha A$ doesn't hold for $\alpha \in\left\{\epsilon_{h}, q_{\delta}, \in_{h} \vee q_{\delta}, \in_{h} \wedge q_{\delta}\right\}$.

Definition 1 A fuzzy subset $A$ on $\mathscr{L}$ is called an $(\alpha, \beta)$-fuzzy filter, if it satisfies, for any $x, y \in L, t, r \in(h, 1]$ and $h<\delta$ :

(F5) $x_{t} \alpha A$ implies $I_{t} \beta A$,

(F6) if $x_{t} \alpha A$ and $(x \rightarrow y)_{r} \alpha A$, then $y_{\min \{t, r\}} \beta A$, where $\alpha, \beta \in\left\{\in_{h}, q_{\delta}, \in_{h} \vee q_{\delta}, \in_{h} \wedge q_{\delta}\right\}$ but $\alpha \neq \in_{h} \wedge q_{\delta}$.

In Definition 1, the case $\alpha=\epsilon_{h} \wedge q_{\delta}$ can be omitted. Since for a fuzzy subset $A$ such that $A(x)<\delta$ for any $x \in L$, and $x_{t} \in_{h} \wedge q_{\delta} A$, we have $A(x) \geq t>h$ and $A(x)+t>2 \delta$. Thus $2 A(x)=A(x)+A(x) \geq A(x)+t>2 \delta$ and so $A(x)>\delta$. Hence $\left\{x_{t} \mid x_{t} \in_{h} \wedge q_{\delta}\right\}=\emptyset$. This explains why $\alpha=\epsilon_{h} \wedge q_{\delta}$ should be omitted in Definition 1.

It isn't difficult to see, any $(\alpha, \beta)$-fuzzy filter on $\mathscr{L}$ must be an $\left(\alpha, \in_{h} \vee q_{\delta}\right)$-fuzzy filter on $\mathscr{L}$. Hence the $\left(\alpha, \in_{h} \vee q_{\delta}\right)$-fuzzy filter plays a central role in the theory of $(\alpha, \beta)$-fuzzy filter. So we only need to study the $\left(\alpha, \in_{h} \vee q_{\delta}\right)$-fuzzy filter.

In Definition 1, we taking $h=0$ and $\delta=0.5$, an $\left(\epsilon_{h}, \in_{h} \vee q_{\delta}\right)$-fuzzy filters will be an $(\in, \in \vee q)$-fuzzy filter. So $\left(\in_{h}, \in_{h} \vee q_{\delta}\right)$ fuzzy filter is a generalization of $(\in, \in \vee q)$-fuzzy filter in (J.M.Zhan,2009).

Example 1 Let $L=\{O, a, b, c, d, I\}$, the Hasse diagram of $L$ be defined as Figure.1 and its implication operator $\rightarrow$ and negation operator' be defined as Table 1 . Then $\mathscr{L}=\left(L, \vee, \wedge,{ }^{\prime}, \rightarrow, O, I\right)$ is a lattice implication algebra.

(1) we define a fuzzy subset $A$ of $\mathscr{L}$

$$
A(x)= \begin{cases}0.7 & x \in\{I, b, c\}, \\ 0.2 & x \in\{O, d, a\} .\end{cases}
$$

It is routine to verify that $A$ is an $\left(\in_{0.3}, \in_{0.3}\right)$-fuzzy filter£

(2) we define a fuzzy subset $B$ of $\mathscr{L}$

$$
B(x)= \begin{cases}0.6 & x=I, \\ 0.7 & x \in\{b, c\}, \\ 0.2 & x \in\{O, d, a\} .\end{cases}
$$

It is routine to verify that $B$ is an $\left(\epsilon_{0.3}, \epsilon_{0.3} \vee q_{0.6}\right)$-fuzzy filter.

Theorem 1 Let $h, \delta \in[0,1], h<\delta$ and $A$ be a fuzzy subset of $\mathscr{L}$. Then $A$ is an $\left(\in_{h}, \in_{h} \vee q_{\delta}\right)$-fuzzy filter if and only $A$ satisfies following two conditions:

(1) $(\forall x \in L)(\max \{A(I), h\} \geq \min \{A(x), \delta\})$,

(2) $(\forall x, y \in L)(\max \{A(y), h\} \geq \min \{A(x), A(x \rightarrow y), \delta\}$.

Proof $(F 5) \Rightarrow(1)$ Assume there exist $x \in L$ such that $\max \{A(I), h\}<\min \{A(x), \delta\}=r$, then $A(x) \geq r>h$ and $r \leq \delta$, hence $x_{r} \in_{h} A$. It follows that $I_{r} \in_{h} \vee q_{\delta} A$ by $F(5)$, we have $A(I) \geq r>h$ or $A(I)+r>2 \delta$. Since $\max \{A(I), h\}<r$, it follows that $A(I)<r$ and $A(I)+r<2 r \leq 2 \delta$, contradiction. Therefore, (1) is valid.

(1) $\Rightarrow F(5)$ Assume (1) holds and $F(5)$ doesn't hold, then there exist $y \in L$ such that $y_{r} \in_{h} A$, but $I_{r} \overline{\epsilon_{h} \vee q_{\delta}} A$, that is $A(I)<r$ and $A(I)+r \leq 2 \delta$, it follows that $A(I)<\delta$, hence $A(I)<\min \{\delta, r\}$. Since $A(y) \geq r>h$, we have

$$
\max \{A(I), h\} \geq \min \{A(y), \delta\}>\min \{\delta, r\} .
$$

It follows that $\min \{\delta, r\}>h$ for $\delta, r>h$, therefore $A(I)>\min \{\delta, r\}$,contradiction. Hence $F(5)$ holds.

$F(6) \Rightarrow(2)$ Assume that there exist $x, y \in L$ such that $\max \{A(y), h\}<\min \{A(x), A(x \rightarrow y)\}=r$, then $A(x) \geq r>h$, $A(x \rightarrow y) \geq r>h, \delta \geq r, A(y)<r$. Therefore $x_{r} \in_{h} A$ and $(x \rightarrow y)_{r} \in_{h} A$. It follows that $y_{r} \in \in_{h} \vee q_{\delta} A$ by $F(6)$, that is $A(y) \geq r>h$ or $A(y)+r>2 \delta$. Since $A(y)<r$, we must have $A(y)+r<r+r=2 r \leq 2 \delta$, which contradicts with $A(y)+r>2 \delta$. Hence (2) holds.

(2) $\Rightarrow F(6)$ Assume that there exist $x, y \in L$ such that $x_{t} \in_{h} A,(x \rightarrow y)_{r} \in_{h} A$, but $y_{\min \{t, r\}} \overline{\epsilon_{h} \vee q_{\delta}} A$, then $A(x) \geq t>$ $h, A(x \rightarrow y) \geq r>h$ but $A(y)<\min \{t, r\}$ and $A(y)+\min \{t, r\} \leq 2 \delta$. Hence $A(y)<\delta$. It follows that $A(y)<\min \{\delta, t, r\}$. We 
have $\max \{A(y), h\} \geq \min \{A(x), A(x \rightarrow y), \delta\} \geq \min \{t, r, \delta\}$, it follows that $\min \{t, r, \delta\}>\max \{A(y), h\} \geq \min \{A(x), A(x \rightarrow$ $y), \delta\} \geq \min \{t, r, \delta\}$, contradiction. Therefore (2) is valid.

Remark From Theorem 1, the Definition of $\left(\epsilon_{h}, \epsilon_{h} \vee q_{\delta}\right)$-fuzzy filter coincident with the concept of fuzzy filter with a threshold $(h, \delta]$ in a lattice implication algebra(J.M.Zhan 2009).

Theorem 2 Let $A$ be an $\left(\in_{h}, \in_{h} \vee q_{\delta}\right)$ - fuzzy filter of $\mathscr{L}$ and $1+h=2 \delta$, then $U\left(A ; h^{>}\right)$is a filter of $\mathscr{L}$, where $h<\delta$ and $U\left(A ; h^{>}\right)=\{x \in L \mid A(x)>h\}$.

Proof Let $A$ be an $\left(\epsilon_{h}, \in_{h} \vee q_{\delta}\right)$-fuzzy filter. Since $x_{A(x)} \in_{h} A$, we have $I_{A(x)} \in_{h} \vee q_{\delta} A$, that is $A(I) \geq A(x)>h$ or $A(I)+A(x)>2 \delta$. Hence $A(I)>h$ or $A(I)>2 \delta-A(x)>2 \delta-1=h$, we must have $I \in U\left(A ; h^{>}\right)$.

Let $x, x \rightarrow y \in U\left(A ; h^{>}\right)$, then $A(x)>h$ and $A(x \rightarrow y)>h$. Since $A$ is an $\left(\in_{h}, \in_{h} \vee q_{\delta}\right)$-fuzzy filter, we have $\max A(y), h \geq$ $\min \{A(x), A(x \rightarrow y)\}, \delta)>\min \{h, \delta\}=\delta$ for any $x, y \in L$ by Theorem 1 . Hence $A(y)>h$, that is $y \in U\left(A ; h^{>}\right)$. It follows that $U\left(A ; h^{>}\right)$is a filter of $\mathscr{L}$.

Let $S$ be a non-empty subset of $\mathscr{L}$, we define a fuzzy subset $A_{h}^{\delta}$ as follows: $A_{h}^{\delta}(x) \geq \delta$ if and only if $x \in S, A_{h}^{\delta}(x)=h$ if and only if $x \notin S$.

Theorem 3 Let $S$ be a non-empty subset of $\mathscr{L}$ and $h, \delta \in[0,1], h<\delta, 1+h=2 \delta$. Then $S$ is a filter of $\mathscr{L}$ if and only if $A_{h}^{\delta}$ is an $\left(\in_{h}, \in_{h} \vee q_{\delta}\right)$-fuzzy filter of $\mathscr{L}$.

Proof Let $S$ be a filter of $\mathscr{L}$ and $x_{t} \in_{h} A_{h}^{\delta}$ for any $x \in L$, then $A_{h}^{\delta}(x) \geq t>h$. It follows that $A_{h}^{\delta}(x) \geq \delta$, then $x \in S$. Since $I \in S$, we have $A_{h}^{\delta}(I) \geq \delta>h$. If $t \leq \delta$, then $A_{h}^{\delta}(I) \geq \delta \geq t>h$, that is $I_{t} \in_{h} A_{h}^{\delta}$. If $t>\delta$, then $A_{h}^{\delta}(I)+t>\delta+\delta=2 \delta$, that is $I_{t} q_{\delta} A_{h}^{\delta}$. Therefore, in any case, we have $I_{t} \in \epsilon_{h} \vee q_{\delta} A_{h}^{\delta}$.

Let $x_{t},(x \rightarrow y)_{r} \in_{h} A_{h}^{\delta}$, then $A_{h}^{\delta}(x) \geq t>h, A_{h}^{\delta}(x \rightarrow y) \geq r>h$. Hence $A_{h}^{\delta}(x) \geq \delta, A_{h}^{\delta}(x \rightarrow y) \geq \delta$, that is $x \in S, x \rightarrow y \in S$. It follows that $y \in S$ for $S$ is filter of $\mathscr{L}$. That is $A_{h}^{\delta}(y) \geq \delta$. If $\min \{t, r\} \leq \delta$, then $A_{h}^{\delta}(y) \geq \delta \geq \min \{t, r\}>h$, it follows that $y_{\min \{t, r\}} \in \in_{h} A_{h}^{\delta}$. If $\min \{t, r\}>\delta$, then $A_{h}^{\delta}(y)+\min \{t, r\}>\delta+\delta=2 \delta$, we have $y_{\min \{t, r\}} q_{\delta} A_{h}^{\delta}$. So $y_{\min \{t, r\}} \in \in_{h} \vee q_{\delta} A_{h}^{\delta}$. Therefore $A_{h}^{\delta}$ is an $\left(\in_{h}, \in_{h} \vee q_{\delta}\right)$-fuzzy filter of $\mathscr{L}$.

Conversely, Assume that $A_{h}^{\delta}$ is an $\left(\epsilon_{h}, \in_{h} \vee q_{\delta}\right)$-fuzzy filter of $\mathscr{L}$. For any $x \in S$, we have $A_{h}^{\delta}(x) \geq \delta>h$, that is $x \in U\left(A_{h}^{\delta} ; h^{>}\right)$, so $S \subseteq U\left(A_{h}^{\delta} ; h^{>}\right)$. If $x \in U\left(A_{h}^{\delta} ; h^{>}\right)$, then $A_{h}^{\delta}(x)>h$, that is $A_{h}^{\delta} \geq \delta$. It follows that $x \in S$. Hence $U\left(A_{h}^{\delta} ; h^{>}\right) \subseteq S$. So $S=U\left(A_{h}^{\delta} ; h^{>}\right)$. Therefore, $S$ is a filter by Theorem 2 .

From the Proof of Theorem 3, it is easy to obtain the corollary 4.

Corollary 4 Let $h, \delta \in[0,1], h<\delta$ and $S$ be a filter of $\mathscr{L}$, then $A_{h}^{\delta}$ is an $\left(q_{\delta}, \epsilon_{h} \vee q_{\delta}\right)$-fuzzy filter of $\mathscr{L}$.

The following propositions are obvious, so the proofs are omitted.

Proposition 5 Let $h, \delta \in[0,1], h<\delta$ and $A$ be a fuzzy subset of $\mathscr{L}$. If $A$ is an $\left(\epsilon_{h} \vee q_{\delta}, \in_{h} \vee q_{\delta}\right)$-fuzzy filter of $\mathscr{L}$, then $A$ is an $\left(\epsilon_{h}, \in_{h} \vee q_{\delta}\right)$-fuzzy filter of $\mathscr{L}$.

The following Example show that the converse of Proposition 5 doesn't hold in general.

Example 2 In Example 1, we define a fuzzy subset $A$ of $\mathscr{L}$

$$
A(x)= \begin{cases}0.6 & x=I, \\ 0.7 & x \in\{b, c\}, \\ 0.3 & x=d, \\ 0.2 & x \in\{O, a\} .\end{cases}
$$

It is routine to verify that $A$ is an $\left(\epsilon_{0.3}, \in_{0.3} \vee q_{0.6}\right)$-fuzzy filter. But $A$ is't an $\left(\epsilon_{0.3} \vee q_{0.6}, \in_{0.3} \vee q_{0.6}\right)$-fuzzy filter, since $d_{0.92} \epsilon_{0.3} \vee q_{0.6} A$ and $(d \rightarrow a)_{0.7} \in_{0.3} \vee q_{0.6} A$, but $a_{m i n\{0.92,0.7\}}=a_{0.7} \overline{\epsilon_{0.3} \vee q_{0.6}} A$.

Combining Theorem 1 and Proposition 5, we have the following corollary:

Corollary 6 Any $\left(\epsilon_{h} \vee q_{\delta}, \in_{h} \vee q_{\delta}\right)$-fuzzy filter of $\mathscr{L}$ satisfies the following conditions:

(1) $(\forall x \in L)(\max \{A(I), h\} \geq \min \{A(x), \delta\})$,

(2) $(\forall x, y \in L)(\max \{A(y), h\} \geq \min \{A(x), A(x \rightarrow y), \delta\}$.

Proposition 7 Let $h<\delta$ and $A$ be a fuzzy subset of $\mathscr{L}$. If $A$ is an $\left(\in_{h}, \epsilon_{h}\right)$-fuzzy filter of $\mathscr{L}$, then $A$ is an $\left(\in_{h}, \in_{h} \vee q_{\delta}\right)$-fuzzy filter of $\mathscr{L}$.

The converse of Proposition 7 doesn't hold in general. For example, $B$ is an $\left(\in_{0.3}, \epsilon_{0.3} \vee q_{0.6}\right)$-fuzzy filter. But $B$ isn't an $\left(\epsilon_{0.3}, \in_{0.3}\right)$-fuzzy filter, since $b_{0.65} \in_{0.3} B$, but $I_{0.65} \overline{\epsilon_{0.3}} B$.

Theorem 8 Let $A$ be a fuzzy subset of $\mathscr{L}$. Then $A$ is an $\left(\epsilon_{h}, \epsilon_{h}\right)$-fuzzy filter if and only if $A$ is a fuzzy filter of $\mathscr{L}$.

Proof Let $A$ be an $\left(\in_{h}, \in_{h}\right)$-fuzzy filter of $\mathscr{L}$. Since $x_{A(x)} \in_{h} A$ for any $x \in L$, we have $I_{A(x)} \in_{h} A$, that is $A(I) \geq A(x)$. 
There is a fact that $x_{A(x)} \in_{h} A,(x \rightarrow y)_{A(x \rightarrow y)} \in_{h} A$, it follows that $y_{\min \{A(x), A(x \rightarrow y)\}} \in_{h} A$, that is $A(y) \geq \min \{A(x), A(x \rightarrow y)\}$. Therefore, $A$ is a fuzzy filter of $\mathscr{L}$.

Conversely, let $x_{t} \in_{h} A$, that is $A(x) \geq t>h$, then $A(I) \geq A(x)$ for any $x \in L$ since $A$ is a fuzzy filter. Therefore, $A(I) \geq A(x) \geq t>h$, that is $I_{t} \in_{h} A$.

Let $x_{t} \in_{h} A,(x \rightarrow y)_{r} \in_{h} A$, then $A(x) \geq t>h$ and $A(x \rightarrow y) \geq r>h$. Since $A$ is a fuzzy filter, we have $A(y) \geq \min \{A(x), A(x \rightarrow y)\} \geq \min \{t, r\}>h$, it follows that $y_{\min \{t, r\}} \in_{h} A$. Therefore $A$ is an $\left(\in_{h}, \in_{h}\right)$-fuzzy filter of $\mathscr{L}$.

Theorem 9 Let $h, \delta \in[0,1], h<\delta$ and $A$ be an $\left(\epsilon_{h}, \epsilon_{h} \vee q_{\delta}\right)$-fuzzy filter of $\mathscr{L}$ and $A(x)<\delta$ for any $x \in L$. Then $A$ is an $\left(\in_{h}, \in_{h}\right)$-fuzzy filter of $\mathscr{L}$.

Proof Let $x \in L$ and $t \in(h, 1]$ such that $x_{t} \in_{h} A$, then $A(x) \geq t>h$. It follows that $\max \{A(I), h\} \geq \min \{A(x), \delta\}=A(x) \geq$ $t>h$. Then $A(x)>h$, We have $A(I) \geq t>h$, that is $I_{t} \in_{h} A$.

Let $x, y \in L$ and $t, r \in(0,1]$ such that $x_{t} \in_{h} A$ and $(x \rightarrow y)_{r} \in_{h} A$. Hence $A(x) \geq t>h$ and $A(x \rightarrow y) \geq r>h$. Since $A$ is an $\left(\in_{h}, \in_{h} \vee q_{\delta}\right)$-fuzzy filter, we have $\max \{A(y), h\} \geq \min \{A(x), A(x \rightarrow y), \delta\}=\min \{A(x), A(x \rightarrow y)\} \geq \min \{t, r\}>h$. It follows that $A(y)>h$. Therefore $A(y) \geq \min \{t, r\}>h$, that is $y_{\min \{t, r\}} \in_{h} A$. We have $A$ is an $\left(\in_{h}, \in_{h}\right)$-fuzzy filter of $\mathscr{L}$.

Let $r \in(h, 1], h<\delta$ and $A$ be fuzzy set of $\mathscr{L}$. We denote

$A_{r}=\left\{x \in L \mid x_{r} \in_{h} A\right\}$,

$A_{r, \delta}=\left\{x \in L \mid x_{r} q_{\delta} A\right\}$,

$\Sigma=\left\{x \in L \mid x_{r} \in_{h} \vee q_{\delta} A\right\}$.

Obviously, $\Sigma=A_{r} \cup A_{r, \delta}$.

Theorem 10 Let $A$ be fuzzy set of $\mathscr{L}$ and $h<\delta$.

(1) $A$ is an $\left(\in_{h}, \in_{h} \vee q_{\delta}\right)$-fuzzy filter if and only $A_{r}(\neq \emptyset)$ is a filter of $\mathscr{L}$ for any $r \in(h, \delta]$.

(2) If $1+h=2 \delta$, then $A$ is an $\left(\epsilon_{h}, \in_{h} \vee q_{\delta}\right)$-fuzzy filter if and only $A_{r, \delta}(\neq \emptyset)$ is a filter of $\mathscr{L}$ for any $r \in(\delta, 1]$.

(3) If $1+h=2 \delta$, then $A$ is an $\left(\in_{h}, \in_{h} \vee q_{\delta}\right)$-fuzzy filter if and only $\sum(\neq \emptyset)$ is a filter of $\mathscr{L}$ for any $r \in(h, 1]$.

Proof (1) The proof is straightforward from the Theorem 2.7 in (J.M.Zhan,2009).

We only to prove (3), (2) can be proved analogously.

Let $A$ be an $\left(\in_{h}, \in_{h} \vee q_{\delta}\right)$-fuzzy filter, for any $r \in(h, 1], x \in \sum$, we have $\max \{A(I), h\} \geq \min \{A(x), \delta\}$. Since $x_{r} \in \in_{h} \vee q_{\delta} A$, it follows that $A(x) \geq r>h$ or $A(x) \geq 2 \delta-r \geq 2 \delta-1=h$. Hence

$$
\max \{A(I), h\} \geq \min \{A(x), \delta\}>\min \{h, \delta\}=h,
$$

we have $A(I)>h$. Therefore $A(I)=\max \{A(I), h\} \geq \min \{A(x), \delta\}$. There are two cases need to be discussed:

Case I: When $r \in(h, \delta$ ], then $2 \delta \geq \delta>r$. We have $A(I) \geq \min \{A(x), \delta\} \geq \min \{r, \delta\}=r>h$ or $A(I) \geq \min \{A(x), \delta\} \geq$ $\min \{\delta, 2 \delta-r\}=\delta \geq r>h$, then $I_{r} \in_{h} A$.

Case II: When $r \in(\delta, 1]$, then $2 \delta<\delta<r$. We have $A(I) \geq \min \{A(x), \delta\} \geq \min \{r, \delta\}=r>2 \delta-r$ or $A(I) \geq \min \{A(x), \delta\} \geq$ $\min \{\delta, 2 \delta-r\}=2 \delta-r$, then $I_{r} q_{\delta} A$.

Therefore, we have $I_{r} \in_{h} \vee q_{\delta} A$ from Case I,II. That is, $I \in \sum$.

Let $x, x \rightarrow y \in \sum$, then $x_{r} \in_{h} \vee q_{\delta} A$ and $(x \rightarrow y)_{r} \in_{h} \vee q_{\delta} A$. There are four cases need to be discussed:

(a) If $x_{r} \in_{h} A$ and $(x \rightarrow y)_{r} \in_{h} A$, then $y_{r} \in \in_{h} \vee q_{\delta} A$, that is $y \in \sum$.

(b) If $x_{r} \in_{h} A$ and $(x \rightarrow y)_{r} q_{\delta} A$, then $A(x) \geq r>h$ and $A(x \rightarrow y)+r>2 \delta$. It follows that $A(x) \geq r>h$ and $A(x \rightarrow y)>2 \delta-r$. Since $A$ is an $\left(\in_{h}, \in_{h} \vee q_{\delta}\right)$-fuzzy filter, we have $\max A(y), h \geq \min \{A(x), A(x \rightarrow y), \delta\}>\min \{r, 2 \delta-r, \delta\}$. If $r \in(h, \delta]$, then $2 \delta-r \geq \delta \geq r$. Therefore $\max A(y), h \geq \min \{A(x), A(x \rightarrow y), \delta\}>\min \{r, 2 \delta-r, \delta\}>r$, that is $A(y) \geq r>h$, so $y_{r} \in h A$. Hence $y_{r} \in_{h} \vee q_{\delta} A$, so $y \in \sum$. If $r \in(\delta, 1]$, then $2 \delta-r<\delta<r$. It follows that $\max \{A(y), h\} \geq \min \{A(x), A(x \rightarrow y), \delta\}>\min \{r, 2 \delta-r, \delta\}=2 \delta-r>2 \delta-1=h$, so $A(y)>h$. Therefore $A(y)=$ $\max A(y), h \geq \min \{A(x), A(x \rightarrow y), \delta\}>\min \{r, 2 \delta-r, \delta\}=2 \delta-r$, so $A(y)+r>2 \delta$. It follows that $y_{r} q_{\delta} A$, of course, $y_{r} \in_{h} \vee q_{\delta} A$, that is $y \in \sum$.

(3) If $x_{r} q_{\delta} A$ and $(x \rightarrow y)_{r} \in_{h} A$, then, similar with proof of (2), we can obtain $y \in \sum$.

(4) If $x_{r} q_{\delta} A$ and $(x \rightarrow y)_{r} q_{\delta} A$, then $A(x)+r>2 \delta$ and $A(x \rightarrow y)+r>2 \delta$, that is $A(x)>2 \delta-r$ and $A(x \rightarrow y)>2 \delta-r$. Since $A$ is an $\left(\epsilon_{h}, \epsilon_{h} \vee q_{\delta}\right)$-fuzzy filter, we have $\max A(y), h \geq \min \{A(x), A(x \rightarrow y), \delta\}>\min \{2 \delta-r, \delta\}$. The remaining discussion is analogous to the (2). We have $y \in \sum$. 
Sum up above, $\sum$ is a filter of $\mathscr{L}$.

Conversely, Let $\sum$ is a filter of $\mathscr{L}$, then $I \in \sum$, that is $I_{r} \in_{h} \vee q_{\delta} A$.

Let $x_{r} \in_{h} A$ and $(x \rightarrow y)_{t} \in_{h} A$, that is $A(x) \geq r>h$ and $A(x \rightarrow y) \geq t>h$. We have $A(x) \geq r \geq \min \{t, r\}>h$ and

$$
A(x \rightarrow y) \geq t \geq \min \{t, r\}>h,
$$

then $x_{\min \{t, r\}} \in_{h} A$ and $(x \rightarrow y)_{\min \{t, r\}} \in_{h} A$. We can obtain $x, x \rightarrow y \in \sum$. Since $\sum$ is a filter, we have $y \in \sum$, that is $y_{\min \{t, r\}} \in_{h} \vee q_{\delta} A$. Therefore $A$ is an $\left(\in_{h}, \in_{h} \vee q_{\delta}\right)$-fuzzy filter of $\mathscr{L}$.

Corollary 11 Let $A$ be fuzzy set of $\mathscr{L}$.

(1) $A$ is an $(\in, \in \vee q)$-fuzzy filter if and only $U(A ; r)(\neq \emptyset)$ is a filter of $\mathscr{L}$ for any $r \in(0,0.5]$, where $U(A ; r)=\left\{x \in L \mid x_{r} \in\right.$ A\}.

(2) $A$ is an $(\in, \in \vee q)$-fuzzy filter if and only $U(A ; r)(\neq \emptyset)$ is a filter of $\mathscr{L}$ for any $r \in(0.5,1]$, where $Q(A ; r)=\left\{x \in L \mid x_{r} q A\right\}$.

(3) $A$ is an $(\in, \in \vee q)$-fuzzy filter if and only $\sum(A ; r)(\neq \emptyset)$ is a filter of $\mathscr{L}$ for any $r \in(0,1]$, where $\sum(A ; r)=\left\{x \in L \mid x_{r} \in\right.$ $\vee q A\}$.

4. $(\bar{\alpha}, \bar{\beta})$-fuzzy filters

Definition 2 A fuzzy subset $A$ on $\mathscr{L}$ is said to be an $(\bar{\alpha}, \bar{\beta})$-fuzzy filter, if it satisfies, for any $x, y \in L, t, r \in(h, 1]$ and $h<\delta$ :

(F7) $I_{t} \bar{\alpha} A$ implies $x_{t} \bar{\beta} A$,

(F8) if $y_{\min \{t, r\}} \bar{\alpha} A$, then $x_{t} \bar{\beta} A$ or $(x \rightarrow y)_{r} \bar{\beta} A$, where $\bar{\alpha}, \bar{\beta} \in\left\{\overline{\epsilon_{h}}, \overline{q_{\delta}}, \overline{\epsilon_{h}} \vee \overline{q_{\delta}}, \overline{\epsilon_{h}} \wedge \overline{q_{\delta}}\right\}$ but $\bar{\alpha} \neq \overline{\epsilon_{h}} \wedge \overline{q_{\delta}}$.

In Definition 2, the case $\bar{\alpha}=\overline{\epsilon_{h}} \wedge \overline{q_{\delta}}$ can be omitted, the same reason with Definition 1 .

Example 3 In Example 1, we define a fuzzy set $A$ as follows:

$$
A(O)=0.4, A(I)=A(b)=A(c)=0.9, A(a)=A(d)=0.5 .
$$

It is routine to verify $A$ is an $\left(\overline{\epsilon_{0.3}}, \overline{\epsilon_{0.3}} \vee \overline{q_{0.6}}\right)$-fuzzy filter of $\mathscr{L}$.

Theorem 12 Let $A$ be a fuzzy subset of $\mathscr{L}$, then $A$ is an $\left(\overline{\epsilon_{h}}, \overline{\epsilon_{h}} \vee \overline{q_{\delta}}\right)$-fuzzy filter if and only if for any $x, y \in L, t, r \in(h, 1]$ and $h<\delta$,

(1) $\max \{A(I), \delta\} \geq A(x)$,

(2) $\max \{A(y), \delta\} \geq \min \{A(x), A(x \rightarrow y)\}$.

Proof Assume that (F7) hold and there exists $x \in L$ such that $\max \{A(I), \delta\}<A(x)=t$. Then $t \in(\delta, 1]$ and $I_{t} \overline{\epsilon_{h}} A$. It follows that $x_{t} \bar{\beta} A$ from (F7). Hence $A(x)<t$ or $A(x)+t \leq 2 \delta$, we have $t \leq \delta$ for $A(x)=t$, contradiction. Therefore, $\max \{A(I), \delta\} \geq A(x),(1)$ is valid.

Assume that there exist $x, y \in L$ such that $\max \{A(y), \delta\}<\min \{A(x), A(x \rightarrow y)\}=t$, then $A(y)<t$ and $t \in(\delta, 1]$. It follows that $y_{t} \overline{\epsilon_{h}} A$. But $x_{t} \in \in_{h} A$ and $(x \rightarrow y)_{t} \in \in_{h} A$. By $(F 8)$, we have $x_{t} \overline{q_{\delta}} A$ or $(x \rightarrow y)_{t} \overline{q_{\delta}} A$. It follows that $A(x) \geq t$ and $A(x)+t \leq 2 \delta, A(x \rightarrow y)=t$ and $A(x \rightarrow y)+t \leq 2 \delta$, we have that $t \geq \delta$, contradiction. Therefore, (2) holds.

Conversely, assume that there exist $x, y \in L$ and $t, r \in(h, 1]$ such that $I_{t} \overline{\epsilon_{h}} A$, but $x_{t} \overline{\overline{\epsilon_{h}}} \vee \overline{q_{\delta}} A$, then $A(I)<t, A(x) \geq t>h$ and $A(x)+t \geq 2 \delta$. Therefore, $A(x) \geq \delta$. Thus $\max \{A(x), \delta\}<\max \{t, \delta\} \geq \max \{A(x), t\}=A(x)$, contradiction. That is, $I_{t} \bar{\alpha} A$ implies $x_{t} \bar{\beta} A$.

Let $y_{\min \{t, r\}} \overline{\epsilon_{h}} A$, then $A(y)<\min \{t, r\}$. There are two cases to be discussed.

(a) If $A(y) \geq \min \{A(x), A(x \rightarrow y)\}$, then $\min \{t, r\}>\min \{A(x), A(x \rightarrow y)\}$. It follows that $A(x)<t$ or $A(x \rightarrow y)<t$, that is, $x_{t} \overline{\epsilon_{h}} A$ or $(x \rightarrow y)_{r} \overline{\epsilon_{h}} A$. Of course, $x_{t} \overline{\epsilon_{h}} \vee \overline{q_{\delta}} A$ or $(x \rightarrow y)_{r} \overline{\epsilon_{h}} \vee \overline{q_{\delta}} A$.

(b) If $A(y)<\min \{A(x), A(x \rightarrow y)\}$, then $\delta \geq \max \{A(y), \delta\} \geq \min \{A(x), A(x \rightarrow y)\}$. Assume that $x_{t} \overline{\overline{\epsilon_{h}} \vee \overline{q_{\delta}}}$ and $(x \rightarrow$ $y)_{r} \overline{\overline{\epsilon_{h}} \vee \overline{q_{\delta}}}$, then $A(x) \geq r$ and $A(x)+r>2 \delta, A(x \rightarrow y) \geq r$ and $A(x \rightarrow y)+r>2 \delta$. It follows that $A(x)>\delta$ and $A(x \rightarrow y)>\delta$. Hence $\min \{A(x), A(x \rightarrow y)\}>\delta$, which contradicts with $\min \{A(x), A(x \rightarrow y)\} \leq \delta$. Therefore, $x_{t} \overline{\epsilon_{h}} \vee \overline{q_{\delta}}$ or $(x \rightarrow y)_{r} \overline{\epsilon_{h}} \vee \overline{q_{\delta}}$.

Theorem 13 Let $h<\delta$ and $A$ be a fuzzy subset of $\mathscr{L}$. Then $U(A ; \alpha), \alpha \in(\delta, 1]$ is a filter of $\mathscr{L}$ if and only if $A$ satisfies

(1) $(\forall x \in L)(\max \{A(I), \delta\}) \geq A(x)$,

(2) $(\forall x, y \in L)(\max \{A(y), \delta\}) \geq \min \{A(x), A(x \rightarrow y)\}$, where $U(A ; \alpha)=\{x \in L \mid A(x) \geq \alpha\}$.

Proof Assume that $U(A ; \alpha), \alpha \in(\delta, 1]$ is a filter of $\mathscr{L}$. If there exists $a \in L$ such that $\max \{A(I), \delta\}<A(a)$, then $A(a) \in(\delta, 1]$ 
and $a \in U(A ; A(a))$, but $A(I)<A(a)$, that is $I \notin U(A ; A(a))$, which contradicts with $U(A ; A(a))$ is a filter. Hence (1) is valid.

Assume that there exist $a, b \in L$ such that $\max \{A(b), \delta\}<\min \{A(a), A(a \rightarrow b)\}=\beta$, then $\beta \in(\delta, 1]$ and $A(a) \geq \beta$, $A(a \rightarrow b) \geq \beta$, that is $a, a \rightarrow b \in U(A ; \beta)$. Since $U(A ; \beta)$ is a filter of $\mathscr{L}$, we have $b \in U(A ; \beta)$, that is $A(b) \geq \beta$. Therefore $\max \{A(b), \delta\} \geq \max \{\beta, \delta\}=\beta$. But $\max \{A(b), \delta\}<\beta$, contradiction. Hence (2) holds.

Conversely, assume that $A$ satisfies (1)(2). Let $\alpha \in(\delta, 1]$, for any $x \in U(A ; \alpha)$, we have $\max \{A(I), \delta\} \geq A(x) \geq \alpha>\delta$. Then $A(I)>\delta$, it follows that $\max \{A(I), \delta\}=A(I) \geq A(x) \geq \alpha$, therefore $I \in U(A ; \alpha)$.

Let $x, y \in L$ and $x, x \rightarrow y \in U(A ; \alpha)$, then $A(x) \geq \alpha, A(x \rightarrow y) \geq \alpha$. Since $\max \{A(y), \delta\}) \geq \min \{A(x), A(x \rightarrow y)\} \geq \alpha>\delta$ for any $x, y \in L$, we have $A(y)>\delta$. Hence $\max \{A(y), \delta\}=A(y) \geq \min \{A(x), A(x \rightarrow y)\} \geq \alpha$, then $y \in U(A ; \alpha)$. Therefore $U(A ; \alpha)(\alpha \in(\delta, 1])$ is a filter of $\mathscr{L}$.

By the Theorem 12 and Theorem 13, we have the following theorem:

Theorem 14 Let $A$ be a fuzzy subset of $\mathscr{L}$. If $A$ is an $\left(\overline{\epsilon_{h}}, \overline{\epsilon_{h}} \vee \overline{q_{\delta}}\right)$-fuzzy filter, then $U(A ; \alpha)$ is a filter of $\mathscr{L}$, where $U(A ; \alpha)=\{x \in L \mid A(x) \geq \alpha\}$ and $\alpha \in(h, \delta]$.

Proposition 15 Let $A$ be a fuzzy subset of $\mathscr{L}$. If $A$ is an $\left(\overline{\epsilon_{h}} \vee \overline{q_{\delta}}, \overline{\epsilon_{h}} \vee \overline{q_{\delta}}\right)$-fuzzy filter, then $A$ is an $\left(\overline{\epsilon_{h}}, \overline{\epsilon_{h}} \vee \overline{q_{\delta}}\right)$-fuzzy filter.

The converse of Proposition 15 doesn't hold in general. For example, in Example $4, A$ is an $\left(\overline{\epsilon_{0.3}}, \overline{\epsilon_{0.3}} \vee \overline{q_{0.6}}\right)$-fuzzy filter, but $A$ isn't an $\left(\overline{\epsilon_{0.3}}, \overline{\epsilon_{0.3}}\right)$-fuzzy filter. Since $O_{0.45} \overline{\epsilon_{0.3}} A$, but $A(b)=0.9>0.45>0.3$ and $A(b \rightarrow O)=A(d)=0.5>0.45>0.3$, we have $b_{0.45} \epsilon_{0.3} A$ and $(b \rightarrow O)_{0.45} \in_{0.3} A$. Therefore, $A$ isn't an $\left(\overline{\epsilon_{0.3}}, \overline{\epsilon_{0.3}}\right)$-fuzzy filter of $\mathscr{L}$.

Proposition 16 Let $A$ be a fuzzy subset of $\mathscr{L}$. If $A$ is an $\left(\overline{\epsilon_{h}}, \overline{\epsilon_{h}}\right)$-fuzzy filter, then $A$ is an $\left(\overline{\epsilon_{h}}, \overline{\epsilon_{h}} \vee \overline{q_{\delta}}\right)$-fuzzy filter.

The converse of Proposition 16 doesn't hold in general. For example, in Example 1, we define a fuzzy subset $B$ as follows: $B(I)=B(b)=B(c)=0.7, B(O)=B(a)=(d)=0.8$. It is routine to verify $B$ is an $\left(\overline{\epsilon_{0.3}}, \overline{\epsilon_{0.3}} \vee \overline{q_{0.6}}\right)$-fuzzy filter. But $B$ isn't an $\left(\overline{\epsilon_{0.3}} \vee \overline{q_{0.6}}, \overline{\epsilon_{0.3}} \vee \overline{q_{0.6}}\right)$-fuzzy filter. Since $B(I)=0.7+0.45<2 \times 0.6$, that is, $I_{0.45} \overline{\epsilon_{0.3}} \vee \overline{q_{0.6}} A$. But $B(d)=0.8$ and

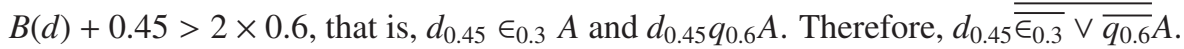

Theorem 17 Let $A$ be a fuzzy subset of $\mathscr{L}$.

(1) $A$ is an $\left(\overline{\epsilon_{h}}, \overline{\epsilon_{h}} \vee \overline{q_{\delta}}\right)$-fuzzy filter if and only if $A_{r}(\neq \emptyset)$ is a filter of $\mathscr{L}$ for any $r \in(\delta, 1]$.

(2) $A$ is an $\left(\overline{\epsilon_{h}}, \overline{\epsilon_{h}} \vee \overline{q_{\delta}}\right)$-fuzzy filter if and only if $A_{r, \delta}(\neq \emptyset)$ is a filter of $\mathscr{L}$ for any $r \in(h, \delta]$.

Proof (1) Let $A$ is an $\left(\overline{\epsilon_{h}}, \overline{\epsilon_{h}} \vee \overline{q_{\delta}}\right)$-fuzzy filter of $\mathscr{L}$ and $I \notin A_{r}$, then $I_{r} \overline{\epsilon_{h}} A$, that is $A(I)<r$. Since $A$ is an $\left(\overline{\epsilon_{h}}, \overline{\epsilon_{h}} \vee \overline{q_{\delta}}\right)$ fuzzy filter of $\mathscr{L}$, we have $\max \{A(I), \delta\} \geq A(x)$ for any $x \in A_{r}$, it follows that $r=\max \{r, \delta\} \geq \max \{A(I), \delta\} \geq A(x)$. Thus $A(x)<r$. But $x \in A_{r}$, that is $A(x) \geq r>h$, contradiction. Therefore $I \in A_{r}$.

Assume that $x, x \rightarrow y \in A_{r}$, that is $A(x) \geq r>h$ and $A(x \rightarrow y) \geq r>h$. Since $A$ is an $\left(\overline{\epsilon_{h}}, \overline{\epsilon_{h}} \vee \overline{q_{\delta}}\right)$-fuzzy filter of $\mathscr{L}$, we have $\max \{A(y), \delta\} \geq \min \{A(x), A(x \rightarrow y)\} \geq r$. It follows that $A(y) \geq r$ for $r \in(\delta, 1]$. Hence $y_{r} \in_{h} A$, that is $y \in A_{r}$. Therefore, $A_{r}$ is a filter of $\mathscr{L}$.

Conversely, suppose that there exists $x \in L$ such that $\max \{A(I), \delta\}<A(x)=r$, then $r \in(\delta, 1], A(I)<r$. That is $I_{r} \overline{\epsilon_{h}} A$. Hence $I \notin A_{r}$, which contradicts with the hypothesis that $A_{r}$ is a filter. Therefore, $\max \{A(I), \delta\} \geq A(x),(F)$. Analogously, we can obtain $\max \{A(y), \delta\} \geq \min \{A(x), A(x \rightarrow y)\}$ for any $x, y \in L$. Thus $A$ is an $\left(\overline{\epsilon_{h}}, \overline{\epsilon_{h}} \vee \overline{q_{\delta}}\right)$-fuzzy filter of $\mathscr{L}$ by Theorem 12 .

(2) Assume that $I \notin A_{r, \delta}$, that is, $I_{r} \overline{q_{\delta}} A$. Thus $A(I)+r \leq 2 \delta$, that is $A(I) \leq 2 \delta-r$. Since $A$ is an $\left(\overline{\epsilon_{h}}, \overline{\epsilon_{h}} \vee \overline{q_{\delta}}\right)$-fuzzy filter of $\mathscr{L}$, then, for any $x \in A_{r, \delta}, \max \{A(I), \delta\} \geq A(x)$. Since $x \in A_{r, \delta}$, then $A(x)+r>2 \delta$. It follows that $\max \{A(I), \delta\} \geq A(x)>2 \delta-r$. By hypothesis $r \in(h, \delta]$, we have $2 \delta-r \geq \delta$. Thus $A(I)>2 \delta-r$, contradiction. Hence $I \in A_{r, \delta}$.

Assume that $x, x \rightarrow y \in A_{r, \delta}$, then $A(x)+r>2 \delta$ and $A(x \rightarrow y)+r>2 \delta$. Since $A$ is an $\left(\overline{\epsilon_{h}}, \overline{\epsilon_{h}} \vee \overline{q_{\delta}}\right)$-fuzzy filter of $\mathscr{L}$, we have $\max \{A(y), \delta\} \geq \min \{A(x), A(x \rightarrow y)\}>2 \delta-r$. Since $2 \delta-r>\delta$ for $r \in(h, \delta]$, it follows that $A(y)>2 \delta-r$, that is $A(y)+r>2 \delta$, thus $y \in A_{r, \delta}$. Therefore, $A_{r \delta}$ is a filter of $\mathscr{L}$.

Conversely, assume that there exist $x, y \in L$ such that $\max \{A(I), \delta\}<A(x)$, then for any $r$ such that $2 \delta-\max \{A(I), \delta\}>$ $r>2 \delta-A(x)$, we have $\min \{2 \delta-A(I), \delta\}>r>2 \delta-A(x)$. Thus $r<\delta, A(I)+r<2 \delta$ and $A(x)+r>2 \delta$. That is, $I_{r} \overline{q_{\delta}} A$, hence $I \notin A_{r, \delta}$, contradiction. Therefore, $\max \{A(I), \delta\} \geq A(x)$. Analogously, we have $\max \{A(y), \delta\} \geq \min \{A(x), A(x \rightarrow y)\}$ for any $x, y \in L$. Thus $A$ is an $\left(\overline{\epsilon_{h}}, \overline{\epsilon_{h}} \vee \overline{q_{\delta}}\right)$-fuzzy filter of $\mathscr{L}$ by Theorem 12.

In Theorem 17, taking $h=0, \delta=0.5$, we have following Corollaries:

Corollary 18 Let $A$ be a fuzzy subset of $\mathscr{L}$.

(1) $A$ is an $\left(\epsilon_{h}, \epsilon_{h} \vee q_{\delta}\right)$-fuzzy filter of $\mathscr{L}$ if and only if $U(A ; r)(\neq \emptyset)$ is a filter of $\mathscr{L}$ for any $r \in(0.5,1]$. 
(2) $A$ is an $\left(\epsilon_{h}, \in_{h} \vee q_{\delta}\right)$-fuzzy filter of $\mathscr{L}$ if and only if $Q(A ; r)(\neq \emptyset)$ is a filter of $\mathscr{L}$ for any $r \in(0,0.5]$.

\section{Conclusion}

In order to research the many-valued logical system whose propositional value is given in a lattice, Xu initiated the concept of lattice implication algebras. Hence for development of this many-valued logical system, it is needed to make clear the structure of lattice implication algebras. In the notion of lattice implication algebra, the partial order can be applied to describe the incomparability and the implication operation can be used to represent the transfer of incomparability. In this paper, we extend the belongs to $(\epsilon)$ relation and quasi-coincidence with $(q)$ relation between fuzzy points and a fuzzy subsets, the concept of $(\alpha, \beta)$-fuzzy filters and $(\bar{\alpha}, \bar{\beta})$-fuzzy filters of lattice implication algebras is introduced and some related properties are investigated. Some equivalent characterizations of these generalized fuzzy filters are derived. Finally, we discussed relations among these generalized fuzzy filters. This idea of this paper can be applied to the fuzzy implicative filter, fuzzy ultra-filter, and so on. We generalized some results in(Y.B.Jum,2007, J.M.Zhan,2009).

Based on these results, we will study primeness and maximality in the $\left(\epsilon_{h}, \in_{h} \vee q_{\delta}\right)$-fuzzy setting. From the view of universal algebra, we will try to investigated the unifying definition of $\left(\epsilon_{h}, \epsilon_{h} \vee q_{\delta}\right)$-fuzzy filter on all logical algebras and study their common properties.

\section{References}

Al. Naraganan, T. Manikantan. (2005). $(\in, \in \vee q)$-fuzzy subnearings and $(\in, \in \vee q)$-fuzzy ideals of near-rings. J.Appl. Math. Computing, 18, 419-430.

B. Davvaz. (2006). ( $\in, \in \vee q)$-fuzzy subnear-rings and ideals. Soft Computing, 10, $206-211$.

J. M. Zhan, Y.B. Jun. (2009). Notes on redefined fuzzy implication filters of lattice implication algebras. Inform. Sci., 179, 3182-3186.

J.M. Zhan, W.A. Dudek, Y.B. Jun. (2009). Interval valued $(\in, \in \vee q)$-fuzzy filter of psedo BL-algebras. Soft Computing, $13,13-21$.

J. Pavelka. (1979). On fuzzy logic. Studia Logica, I,II,III,£eit.Math. Logik u. Grundl.Math., 2545-52, 119-134, 447-464.

J. Rachuånek, D. $\breve{S}$ alounová. (2008). Fuzzy filers and fuzzy prime filters of bounded $R \ell$-monoids and pseudo BLalgebras. Inform. Sci., 178, 3474-3481.

J. Zhan, W.A. Dudek. (2007). Fuzzy $h$-ideals of herings. Inform.Sci., 177, 876-886.

L.A.Zadeh. (1965). Fuzzy set. Inform. Sci., 8, 338-353.

P.M. Pu, Y. M. Liu. (1980). Fuzzy topology I, Neighborhood structure of a fuzzy point and Moore-Smith convergence. J.Math. Anal.Appl., 76, 571-599.

V. Novak. (1982). First order fuzzy logic. Studia Logica, 46(1), 87-109.

Y. B. Jun, S.Z. Song. (2002). On fuzzy implicative filters of lattice implication algebras. J. Fuzzy Math., 10(4), 893-900.

Y. B. Jun, Y. Xu, J. Ma. (2007). Redefined fuzzy implication filters Inform. Sci., 177, 1422-1429.

Y. B. Jun. (2001). Fuzzy positive implicative and fuzzy associative filters of lattice implication algebras. Fuzzy Sets Syst., 121, 353-357.

Y. B. Jun. (2001). The prime filters theorem of lattice implication algebras. Int. J. Math. Sci., 25, 185-192.

Y. Xu, D. Ruan, K.Y. Qin, et al. (2003). Lattice-valued logic-an alternative approach to treat fuzziness and incomparability, Springer-Verlag, Berlin.

Y. Xu, K.Y. Qin. (1993). On filters of lattice implication algebras. J.Fuzzy Math., 2, 251-260.

Y. Xu and K.Y. Qin. (1992). Lattice H implication algebras and lattice implication algebra classes. J. Hebei Mining and Civil Engineering Institute, 3, 139-143.

Y. Xu. (1993). Lattice implication algebra. J.Southwest Jiaotong Univ., 28(1), 20-27.

Resonfeld. (1971). Fuzzy subgroups. J. Math. Anal. Appl., 35, 512-517.

S.K. Bhakat, P. Das. (1996). ( $\in, \in \vee q)$-fuzzy subgroups. Fuzzy Sets Syst., 80, 359-368.

S.K. Bhakat. (1999). ( $\in \vee q)$-level subset. Fuzzy Sets Syst., 103, 529-533.

S.K. Bhakat. (2000). ( $\in, \in \vee q)$-fuzzy normal, quasinormal and maximal subgroups.Fuzzy Sets Syst., 112, 299-312.

W.A. Dudek, M. Shabir, M. Irfan Ali. (2009). ( $\alpha, \beta$ )-fuzzy ideals of hemirings. Comp.Math. Appl., 58, 310-321. 
Table 1. Operators ' and $\rightarrow$ in $\mathscr{L}$

\begin{tabular}{ccccccccc}
\hline$x$ & $x^{\prime}$ & $\rightarrow$ & $O$ & $a$ & $b$ & $c$ & $d$ & $I$ \\
\hline$O$ & $I$ & $O$ & $I$ & $I$ & $I$ & $I$ & $I$ & $I$ \\
$a$ & $c$ & $a$ & $c$ & $I$ & $b$ & $c$ & $b$ & $I$ \\
$b$ & $d$ & $b$ & $d$ & $a$ & $I$ & $b$ & $a$ & $I$ \\
$c$ & $a$ & $c$ & $a$ & $a$ & $I$ & $I$ & $a$ & $I$ \\
$d$ & $b$ & $d$ & $b$ & $I$ & $I$ & $b$ & $I$ & $I$ \\
$I$ & $O$ & $I$ & $O$ & $a$ & $b$ & $c$ & $d$ & $I$ \\
\hline
\end{tabular}

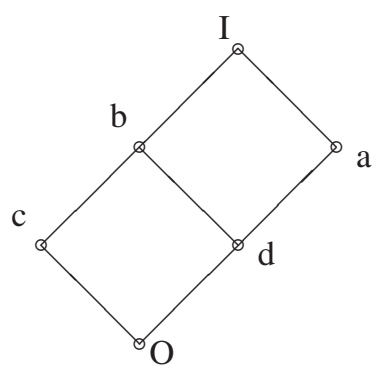

Figure 1. Hasse Diagram of $L=\{O, a, b, c, d, I\}$ 